\title{
Evaluación de citotoxicidad del isotiocianato de bencilo de Troapeolum majus L. en líneas tumorales de mama
}

\section{Cytotoxicity assessment of benzyl isotiocyanate from Troapeolum majus L. breast tumor cell lines}

\author{
Espinoza Silva, C.; Quispe Solano, M.; \\ Véliz Sedano, N.; Baños Medina, L.; Vásquez Porras, V. \\ Facultad de Ingeniería en Industrias Alimentarias / Universidad Nacional del Centro del Perú \\ Email: cespinoza@uncp.edu.pe
}

\section{Resumen}

El mastuerzo (Tropaeolum majus L.) es una planta originaria de Perú, México y partes de América Central; en el Perú, era usada desde tiempos muy antiguos, por nuestros antepasados, para combatir distintas enfermedades. Entre sus componentes se encuentra la glucotropaeolina, que, al ser hidrolizado, forma el bencil isotiocianato (BIT) que fomenta la apoptosis celular. Debido a ello, se tuvo como objetivo; primero, evaluar el efecto citotóxico del isotiocianato de bencilo del mastuerzo expuestas a microgravedad sobre el cultivo in vitro de la línea tumoral MCF-7 de cáncer de mama. Se utilizaron extractos de isotiocianato de bencilo de mastuerzo de la planta cultivada en forma natural (T1) y; la segunda, expuesta a microgravedad en la etapa de germinación (T2), comparándose con el producto comercial (BI), la viabilidad celular se efectuó a través de la técnica del MTT realizándose diluciones de 0, 20, 40, 60, 80 y 100 uM del extracto expuesto al cultivo de células tumorales de tipo MCF-7 cáncer de mama por un tiempo de 12, 24, 48, y 60 horas lecturándose en un lector de placas de ELISA a $570 \mathrm{~nm}$. Se obtuvo una $\mathrm{DL}_{50}$ entre 22,56 \pm 3,24 a 19,97 $\pm 2,52$ a las 60 horas, valores similares a las 48 horas comparadas con el control benzil de isiocianato (BI).

En conclusión, no se evidencia diferencia en el efecto de la dosis letal media de todos los tratamientos en las 48 y 60 horas de incubación frente al benzil isiocianato (BI). El efecto máximo de inhibición en todas las concentraciones es apreciable a las 60 horas de incubación, afirmándose que no existe una diferencia significativa de sus actividades biológicas entre T1 y T2.

\section{Abstract}

The mastuerzo (Tropaeolum majus L.) is a plant native to Peru, Mexico and parts of Central America. In Peru, It was used since ancient times by our ancestors to combat different diseases. Its components include glucotropaeoline, which when hydrolyzed forms benzyl isothiocyanate (BIT) that promotes cellular apoptosis. Due to this, the aim of the work is to evaluate the cytotoxic effect of benzyl isothiocyanate of mastuerzo exposed to microgravity on the in vitro crop of the MCF7 breast cancer cell line. Extracts of bencil isiothiocyanate from the naturally cultivaded plant were used (T1) and the second were exposed to microgravity at the germination stage (T2), comparing with the commercial product (BI), the cell viability was carried out through the MTT technique making dilutions of 0 , $20,40,60,80$ and $100 \mathrm{uM}$ of the extract exposed to tumor cell culture of type MCF-7 breast cancer for a time of $12,24,48$, and 60 hours reading in an ELISA plate reader at $570 \mathrm{~nm}$. Obtaining an LD50 between $22,56 \pm 3,24$ and 19,97 $\pm 2,52$ at 60 hours, values similar to 48 hours compared to the Benzil control of isiocyanate (BI).

In conclusion, no difference were observed between in the effect of the average lethal dose of all treatments in the 48 and 60 hours of incubation an benzyl isiocyanate (BI). The maximum effect of inhibition in all concentrations is appreciable at 60 hours of incubation, stating that there is no significant difference in the biological activities between $\mathrm{T} 1$ and T2.

Keywords: cytotoxicity, MCF-7, breast cancer, benzyl isiocyanate mama, benzil isiocianato 


\section{Introducción}

El mastuerzo (Tropaeolum majus L.) es una planta originaria de Perú, México y partes de América Central. En el Perú, es considerada como una maleza; sin embargo, estudios recientes han confirmado sus bondades médicas, las que se conocen de forma tradicional y que fueron adquiridas por conocimientos ancestrales, se ha demostrado la bondad del mastuerzo como diurético (Gasparotto et al., 2011); así mismo, tiene una acción antimicrobial y antiflamatorio (Butnariu y Bostan, 2011). En este vegetal se han identificado varios componentes bioquímicos, entre los más importantes es el isotiocianato de bencilo, este componente bioquímico, es valorado debido a su capacidad inhibitoria contra las neoplasias cuando se administran después de la exposición al agente carcinógeno (Wattenberg, 1981), fomentando la apoptosis o muerte celular programada en células cancerosas de la mama y ovarios, sin afectar a las células sanas, algo que la quimioterapia no puede garantizar.

La quimio prevención del cáncer se basa en el uso de medicamentos, vitaminas u otros productos químicos, naturales o sintéticos. Su administración tiene como fin favorecer la prevención o retrasar la carcinogénesis, evitando así, la aparición y/o el desarrollo de algunos tipos de cáncer concretos. (Pérez \& Martin, 1998).

El cáncer de mama es el tumor más frecuente en mujeres en países desarrollados. Los expertos señalan, que la aparición del cáncer de mama está estrechamente relacionada con algunos factores de riesgo. Hay evidencias que lo relacionan con la edad, la genética y los factores reproductivos; es decir, el aumento a la exposición a estrógenos endógenos (factores hormonales), la densidad mamaria, la obesidad, las radiaciones ionizantes, la inactividad física, el consumo de alcohol y tabaco, así como el padecimiento de un cáncer de mama anterior. Son importantes factores a tener en cuenta, al ser muchos de ellos modificables y sólo requieren un cambio en el estilo de vida. (Pacheco et al., 2000).

Desde hace décadas se han venido usando líneas cancerígenas como modelos para el estudio y desarrollo de terapias antitumorales. El uso de estas líneas celulares presenta una serie de ventajas, como la homogeneidad de la muestra y el control de las condiciones ambientales (Caba, 2006).

La línea celular MCF-7, fue aislada de una mujer en 1970 y consolidada por Herbert Soule en 1973, consiguiendo así, que este tipo de células pudieran mantenerse en cultivo celular de forma permanente. Esta línea celular fue depositada por C.M. McGrath en ATCC; desde entonces, es una de las líneas más utilizadas para estudios experimentales de cáncer de mama. Se han conseguido diversas variedades de dichas células con diferentes características, aunque pertenecen a la misma línea. (Gooch \& Yee, 1999).
La presente investigación, permite establecer y evaluar in vitro la citotoxicidad del extracto de isotiocianato de bencilo de hojas de Tropaeolum majus en líneas celulares de cáncer de mama.

\section{Métodos y materiales}

\section{Extractos}

Se utilizaron extractos de benzil isotiocionato obtenida de la oleorresina de hojas de mastuerzo (Tropaeolum majus L.) a partir de la planta cultivadas en condiciones normales (T1) y plantas sometidas a microgravedad en la etapa de germinación (T2). La extracción se realizó con $\mathrm{CO}_{2}$ supercrítico a una presión de 200 Bar y $70^{\circ} \mathrm{C}$ presentando $113,615 \pm 0,03 \mathrm{mg}$ de BIT / $100 \mathrm{~g}$ de materia seca. Las que se trabajaron en el Laboratorio de Productos Naturales y Bioactivos de la Facultad de Ingeniería en Industrias Alimentarias de la Universidad Nacional del Centro del Perú - Estación Experimental del Mantaro.

\section{Línea celular}

Esta provino del American Type Culture Collection (ATCC): MCF-7 (adenocarcinoma de mama humano), adquiridas por la empresa Merck del Perú.

Para la variable seguridad alimentaria, se utilizó la Escala de la Inseguridad Alimentaria - Hogar (acrónimo en inglés: FIES-SM), formulada por la Organización de las Naciones Unidas para la Agricultura y Alimentación (acrónimo en inglés - FAO), el cuestionario contiene 8 ítems, los cuales se analizaron en conjunto, mostrando una buena fiabilidad. (FAO, 2014).

\section{Cultivo celular}

Las líneas MCF-7 fueron cultivadas y mantenidas en crecimiento logarítmico en el medio de cultivo RPMI 1640 , suplementado con 7,5\% de suero bovino fetal y $50 \mu \mathrm{g} / \mathrm{mL}$ de gentamicina y mantenida a una temperatura de $37{ }^{\circ} \mathrm{C}$, en un ambiente húmedo, con $95 \%$ de aire y $5 \%$ de $\mathrm{CO}_{2}$. Para resuspender la línea celular, se lavó la monocapa de células con 3 x $4 \mathrm{ml}$ de la solución de Hanks sin Ca-Mg; luego, se agregó $1 \mathrm{ml}$ de la solución de tripsina-EDTA, que después de 10 segundos fue eliminada. Luego se incubó el frasco invertido Flask de $75 \mathrm{cc} 3$ por 8 minutos a $37^{\circ} \mathrm{C}$, al término de los cuales, los cultivos fueron resuspendidos en $2 \mathrm{ml}$ de cada medio de cultivo.

Las células fueron sembradas en una placa de 96 pocillos de fondo plano pretratados para cultivos celular (marca Coaster), con una densidad de 5 x 103 células en condiciones de cultivo, a las 48 horas se observó una confluencia total en cada pocillo.

\section{Ensayo MTT y viabilidad celular}

Con la finalidad de observar el efecto citotóxico del benzol isotiocionato, se utilizó la sustancia comercial de la marca Sigma ${ }^{\circledR}$ versus al extracto obtenido de las 
hojas Tropaeolum majus L. no expuestas a microgravedad (T1) y a la expuestas a microgravedad (T2) (Gamarra \& Ramos, 2017) (Almidón, 2017), sobre la línea celular MCF-7. Para el ensayo de bromuro de 3-(4,5- dimetiltiazol-2-ilo)-2,5-difeniltetrazol (MTT): Se preparó una solución de 100 uM de cada una de las sustancias señaladas utilizando como diluyente 0,02 \% DMSO en medio RPMI - 1640 Sigma ${ }^{\circledR}$ libre de suero fetal de bovino, esta solución fue filtrada a través de una membrana de 0,22um. A partir de esta solución, se prepararon soluciones de: 0, 20, 40, 60, 80 y 100 uM de cada una de las sustancias.

Las células fueron expuestas por un lapso de tiempo de 12, 24, 48, y 60 horas respectivamente, siendo incubadas con el medio de cultivo RPMI - 1640, sin suero fetal bovino, y a $37{ }^{\circ} \mathrm{C}$ con $5 \% \mathrm{CO}_{2}$. Al término de cada incubación, se procedió a colocar 50 ul del reactivo de bromuro de 3-(4,5-dimetiltiazol-2-ilo)-2,5-dife-

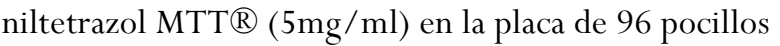
(marca Coaster ${ }^{\circledR}$ ) dejándose incubar por 4 horas a 37 ${ }^{\circ} \mathrm{C}$ y $5 \%$ de $\mathrm{CO}_{2}$, luego se removió la totalidad del líquido y se añadió 150 ul de DMSO e inmediatamente se procedió a realizar la lectura en un lector de placas de ELISA (marca BIOTEK $囚$ model Elx 800) a $570 \mathrm{~nm}$. Finalmente, la actividad metabólica se calculó utilizando la siguiente fórmula:

$\%$ Mortalidad $=\frac{(\text { absMuestra }- \text { absBlanco }) \times 100}{(\text { absControl }- \text { absBlanco })}$

\section{Análisis de datos}

Los resultados obtenidos, fueron analizados mediante el Software - "ED50plus v1.0” para windows y las figuras fueron realizados utilizando el software PRISM 7.

Los análisis estadísticos fueron procesados en el paquete estadístico SPSS_Versión 23 para windows. Las medias de los porcentajes de mortalidad se compararon utilizando el análisis de varianza de una sola vía (ANOVA) junto al post-hoc de TUKEY teniendo como valor discriminatorio de $\mathrm{p}<0,05$ con un nivel de confianza del $95 \%$.

\section{Resultados y discusión}

Los resultados muestran los porcentajes de mortalidad de las células MCF-7 por acción del compuesto activo isotiocianato de bencilo del tratamiento con BI (Benzil de isotiocianato), frente a los tratamientos con los extractos del T1 (extracto de BI obtenidas del vegetal en condiciones normales) y T2 (extractos de BI obtenidas del vegetal llevada a microgravedad en la etapa de germinación). En la Figura 1, se observa que el mayor porcentaje de inhibición se tiene a las 60 horas con una DL50 21,83 $\pm 2,47$ y a su vez se tiene el mismo comportamiento a las 24 y 48 horas con el BI (tratamiento testigo).

\section{Figura 1}

Actividad inhibitoria expresada en el porcentaje de mortalidad de las células MCF-7 como respuesta a las diferentes dosis (20, 40, 60, 80 y $100 \mathrm{uM}) \mathrm{del}$ benzil de isotiocianato (BI) en diferentes tiempos

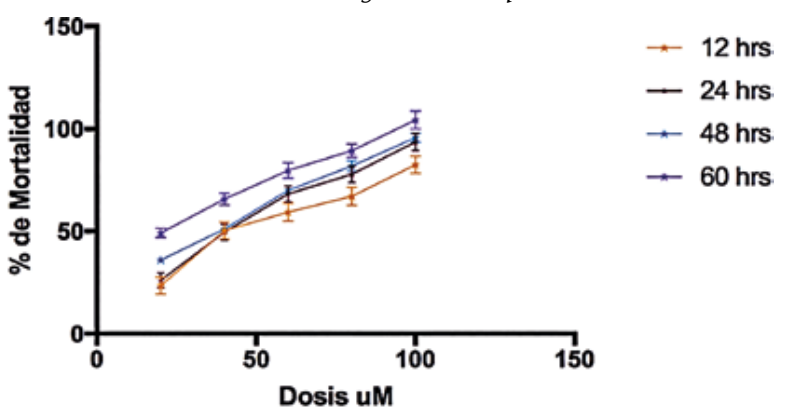

Con respecto a la Figura 2, se puede observar que el tratamiento $\mathrm{T} 1$ presenta una misma tendencia de inhibición dentro de las 24, 48 y 60 horas, con un $\mathrm{DL}_{50}$ máximo de 22,56 $\pm 3,24$.

\section{Figura 2}

Actividad inhibitoria expresada en el porcentaje de mortalidad de las células MCF-7 como respuesta a las diferentes dosis (20,40, 60, 80 y $100 \mathrm{uM})$ del tratamiento 1 (extracto de BI obtenidas del vegetal en condiciones normales) en diferentes tiempos

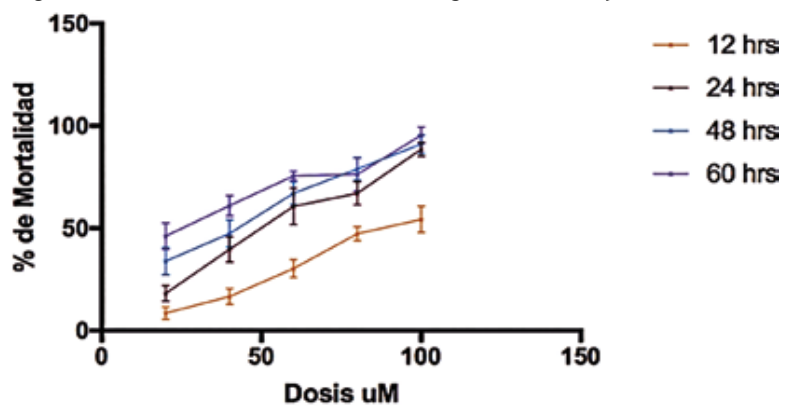

Asimismo, en la Figura 3, se muestra que el tratamiento T2, presenta una tendencia a inhibición parecida dentro de las 12, 24 y 48 horas; además, presenta un ligero $\mathrm{DL}_{50}$ mayor $19,97 \pm 2,52$ a las 60 horas con respecto a las demás concentraciones.

\section{Figura 3.}

Actividad inhibitoria expresada en el porcentaje de mortalidad de las células MCF-7 como respuesta a las diferentes dosis (20, 40, 60, 80 y $100 \mathrm{uM}) \mathrm{del}$ tratamiento 2 (extractos de BI obtenidas del vegetal llevada a microgravedad en la etapa de germinación) en diferentes tiempos

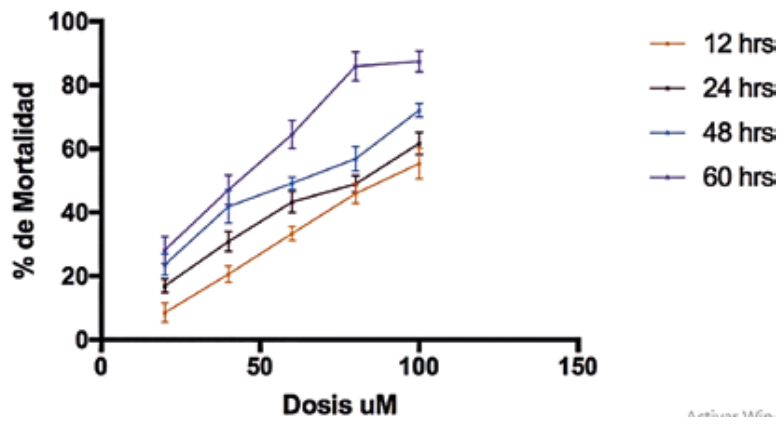


Para comprobar la inhibición del compuesto activo en un periodo extenso, se realizó una incubación en cuatro tiempos, 12, 24, 48 y 60 horas. El valor de Dosis Letal media $50\left(\mathrm{DL}_{50}\right)$ fue comparado entre los tratamientos notándose que en el tiempo de 60 horas de incubación se da el mayor porcentaje de inhibición sin una diferencia significativa entre los tratamientos (Tabla 1).

\section{Tabla 1}

Actividad inhibitoria, expresada en el DL50 de los tratamientos benzil de isotiocianato (BI), tratamiento 1 y tratamiento 2 en la línea de cáncer MCF-7 a diferentes tiempos de incubación

\begin{tabular}{crrr}
\hline $\begin{array}{c}\text { Tiempo de } \\
\text { incubación DL50 BI }(\boldsymbol{\mu M}) \\
\text { (horas) }\end{array}$ & DL50 T1 $(\boldsymbol{\mu M})$ & DL50 T2 $(\boldsymbol{\mu M})$ \\
\hline 12 & $37,30^{\mathrm{a}} \pm 3,50$ & $52,86^{\mathrm{b}} \pm 2,76$ & $51,43^{\mathrm{b}} \pm 1,00$ \\
24 & $41,38^{\mathrm{ab}} \pm 1,19$ & $44,61^{\mathrm{b}} \pm 1,94$ & $39,87^{\mathrm{a}} \pm 0,58$ \\
48 & $34,67^{\mathrm{a}} \pm 1,04$ & $34,87^{\mathrm{a}} \pm 2,63$ & $35,90^{\mathrm{a}} \pm 3,86$ \\
60 & $21,83^{\mathrm{a}} \pm 2,47$ & $22,56^{\mathrm{a}} \pm 3,24$ & $19,97^{\mathrm{a}} \pm 2,52$ \\
\hline
\end{tabular}

Nota: La data expresada corresponde a la media \pm DS de 3 repeticiones de experimento, las letras diferentes evidencian diferencia significativa un nivel de significación del $95 \%$.

A su vez, se puede observar que se evidencia una diferencia de los tratamientos a las 12 y 24 horas de incubación, donde el control benzil de isotiocianato (BI) muestra una mayor actividad inhibitoria, alcanzando una media de 37.3 \pm 3.50 a las 12 horas. Finalmente, se puede apreciar que no hay una diferencia en el efecto de la dosis letal media de los diferentes tratamientos a los 48 y 60 horas de incubación entre elT1,T2 y tratamiento control (Benzil de isotiocianato).

Estos resultados podrían indicar que las células cancerígenas presentan un mecanismo de absorción rápida del compuesto benzil de isotiocianato (BI) en las primeras 12 horas, y que todas las concentraciones tienen una actividad inhibitoria independiente al tratamiento. La farmacocinética del compuesto, indica que se presenta una acción inhibitoria eficaz a partir de las 60 horas de exposición en todos los tratamientos, ya que se tiene como requisito que para un DL50 eficaz anticancerígeno, los valores deben ser menores a 20 uM (Figura 4).

\section{Figura 4}

Actividad inhibitoria expresada en el DL50 de los tratamientos Benzil de isiocianato (BI), tratamiento 1 y tratamiento 2 en la línea de cáncer MCF-7 a los diferentes tiempos de incubación

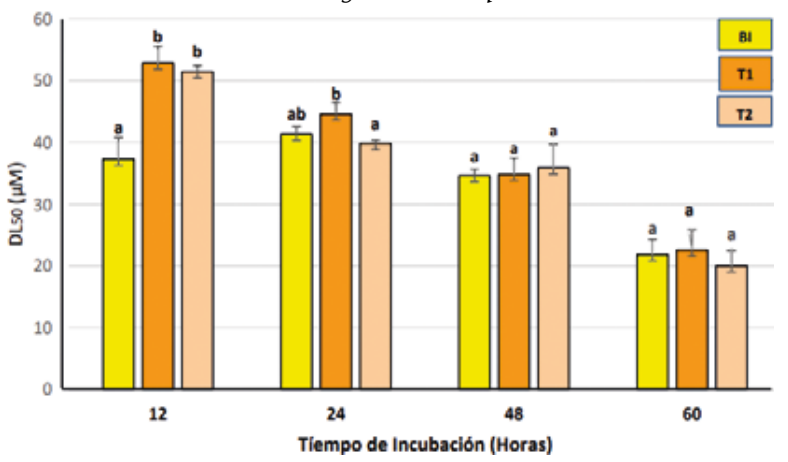

Se evidencia que los extractos de benzil isotiocianato de bencilo, extraidas del mastuerzo (Tropaeolumn majus L.) obtenidas en condición normal y en microgravedad frente a un control de BIT, presentan un incremento de la mortalidad de células cancerígenas proporcional al aumento de la concentración, estableciéndose la línea celular MCF-7 (adenocarcinoma de mama humano).

$\mathrm{Al}$ respecto, Spiridon et al. (2003) mencionan que las propiedades anticancerígenas in vitro de BITC frente a una variedad de líneas de células tumorales humanas y murinas han sido estudiadas por dos métodos independientes: ensayos clonogénicos y cunting de células, esta también se observa a través del método MTT empleado en la investigación, en la que se evidencia que los extractos fitoquímicos contra el cáncer, tomados del mastuerzo, actúan como potentes antioxidantes o antagonistas de los radicales libres y de esta manera minimizan el daño al DNA, previniendo el cáncer (Madhuri \& Pandey, 2009), cumpliendo un rol preventivo en el cáncer a través de sus efectos en la transducción de señales en la proliferación celular (Rajkapoor et al., 2004).

Los isotiocianatos pertenecen a los agentes quimiopreventivos más efectivos, existe una diversidad de isotiocianatos que previenen el cáncer de diferentes tejidos, incluyendo el de pulmón, glándula mamaria, esófago, hígado, intestino delgado, colon y vesícula biliar. En la investigación, se demuestra que el BIT extraido del Tropaeolumn majus L. presenta una actividad citotóxica frente a la línea celular MCF-7 induciendo la apoptosis celular como lo reportado por Lykkesfeldt \& Lindberg (1993). Khan (2013) señala que las líneas celulares de mama normal y tumoral, han sido el modelo más ampliamente usado para investigar cómo se desregulan la proliferación, la apoptosis y la migración en el desarrollo del cáncer de mama a través de estudios in vitro y, que su estudio deriva en una complejidad y heterogeneidad. No es esperable que un único modelo represente todas las variables de la enfermedad (Vargo-Gogola y Rosen, 2007; Rosen, Ashurst y Chap, 2010 mencionados por Siabatto, 2015).

Darwish et al. (2018) evaluaron diferentes partes (flores, tallos y hojas) de las plantas Alpinia officinarum, Achillea millefolium, Colchicum autumnale, Chrysanthemum morifolium, Humulus lupulus, Matricaria chamomilla, Tilia tomentosa, Rosa damascena, Vitexagnus-castus y Silybum marianum sobre células cancerosas A549 y Hep G2 presentando actividad citotóxica IC50 $((\mu \mathrm{g} / \mathrm{ml})$ con valores que van desde $3,66 \pm 1,52$ a 234,6 \pm 7.84 para la primera; y, 7,33 $\pm 3,51$ a 391,6 $\pm 1,45$ para la segunda, los valores obtenidos en la investigación se encuentran por debajo de los máximos obtenidos por los autores, evidenciando que el BIT proveniente de las hojas del mastuerzo presentan una promisoria actividad anticancerígena como lo reflejan los valores $\mathrm{DL}_{50}$ en la línea celular MCF-7 frente a un control BIT. 


\section{Conclusiones}

- El efecto de inhibición mínimo de todas las concentraciones se aprecia a las 12 horas, habiendo una diferencia significativa con respecto a los demás tratamientos de benzil de isotiocianato $\left(\mathrm{DL}_{50} 37,30 \mu \mathrm{M}\right)$. El efecto máximo de inhibición de todas las concentraciones es apreciable a las 60 horas de incubación con una dosis letal media (DL50 $\leq 22,56 \mu \mathrm{M})$. No se evidencia una diferencia significativa entre el tratamiento 1 (T1) y el tratamiento 2 (T2) en los tiempos de incubación de 48 y 60 horas con respecto al BIT control.

\section{Referencias bibliográficas}

Albini, A.; Pennesi, G.; Donatelli, F.; Cammarota, R.; De Flora, S. and Noonan, D. M. (2010). Cardiotoxicity of anticancer drugs: the need for cardio-oncology and cardiooncological prevention. Journal of the National Cancer Institute, vol. 102, no. 1, pp. 14-25.

Almidón, H. (2017). Efecto de la microgravedad en tres fases fenológicas del mastuerzo (Tropaeolum majus L.), Tesis de Grado. Disertación. Universidad Nacional del Centro, Huancayo.

Bao, L.; Matsumura, Y.; Baban, D.; Sun, Y. \& Tarin, D. (1994). Effects of inoculation site and Matrigel on growth and metastasis of human breast cancer cells. British Journal of Cancer, 70(2), 228-232.

Butnariu, M. \& Bostan, C. (2011). Antimicrobial and anti-inflammatory activities of the volatile oil compounds from Tropaeolum majus L. (Nasturtium). African Journal of Biotechnology 10(31):5900-5909. DOI: https://doi.org/10.5897/AJB11.264.

Caba, O. (2006). Potencial terapéutico de nuevos fármacos antitumorales. Estudio sobre líneas epiteliales. PhD. Disertante. Universidad de Granada. España.

Darwish, A. G. G.; Samy, M. N.; Sugimoto, S.; Matsunami, K. \& Otsuka, H. (2018). Componente principal y análisis del mapa de calor de las actividades biológicas para algunas plantas medicinales seleccionadas. Acad. J. Med. Plantas 6 (6): 101-113.

Gamarra, F. \& Ramos, M. (2017). Extracción por diclorometano y CO2 supercrítico de isotiocianato de bencilo a partir de hojas de mastuerzo (Tropaeolum majus L.), Tesis de Grado. Disertación. Universidad Nacional del Centro, Huancayo.

Gasparotto Junior, A.; Gasparotto, F. M.; Boffo, M. A.; Lourenço, E. L. B.; Stefanello, M. É. A.; Salvador, M. J.; Da Silva-Santos, J. E.; Marques, M. C. A. \& Kassuya, C.A. L. (2011). Diuretic and potassium-sparing effect of isoquercitrin-An active flavonoid of Tropaeolum majus L. Journal of Ethnopharmacology 134(2):210-215. DOI: https://doi.org/10.1016/j.jep.2010.12.009.
Gooch, J. L. \& Yee, D. (1999). Strain-specific differences in formation of apoptotic DNA ladders in MCF-7 breast cancer cells. Cancer Letters, 144: 31 - 37.

Khan, N. J. (2013). Cell Lines: An in vitro. Model to study breast cancer. International Journal of Innovative Research in Science, Engineering and Technology. 2 (12), 7214-7219.

Lykkesfeld, J. \& Lindberg, M. B. (1993). Synthesis of benzylglucosinolate in Tropaeolum majus L. Plant Biochemestry Laboratory. Recuperado el 12 de diciembre, 2016 de maltawildplants.com/TROP/Docs/TPLMJ/TRPMJSynthesis_Benzylglucosinolate.pdf

Madhuri, S. \& Pandey, G. (2009). Some anticancer medicinal plants of foreign origin. Current Science 96 (6): 779-783.

Michalopoulos, G.K. 2007. Liver regeneration. J Cell Physiol 213(2): 286-300.

Mosmann, T. (1983). Rapid colorimetric assay for cellular growth and survival: application to proliferation and cytotoxicity assays. J. Immunol Methods. 16; 65(1- 2):55-63.

Pacheco, C.; Tejada, A. \& Moreno, L. (2000). Nuestra experiencia con el tratamiento conservador del cáncer de mama. En: Pérez López F.R., editors. Cáncer de mama: biología, diagnóstico y tratamiento. 1a ed. Zaragoza. SEISGE.P.175- 186.

Pérez, P. \& Martín, M. (1998). Tratamiento sistémico adyuvante en cáncer de mama precoz. En: Díaz-Rubio E, Escudero M, editors. Cáncer de mama. 1a ed. Madrid: International Marketig \& Communications, S.A. P. 141-155.

Rajkapoor B.; Jayakar, B. \& Murugesh, N. (2004). Antitumor activity of indigofera aspalathoides on ehrlich ascites carcinoma on mice. Indian Journal of Pharmacology 36(1): 38-40.

Siabatto, H. (2015). Análisis de los cambios en la expresión génica inducidos por el tratamiento con pseudoterosina en la línea celular de cáncer de mama MDA-MB231 (Tesis inédita para optar el grado de doctor) Universidad Nacional de Colombia

Spiridon, E. \& Barberaki, M. (2003). Plants that fight cancer. Editorial Taylor Francis Group. London and New York. (pag 187).

Tari, A. M. \& Simeone, A. (2005). Cyclooxygenase-2 protein reduces tamoxifen and $N$-(4- hydroxyphenyl) retinamide inhibitory effects in breast cancer cells. Lab Invest 85(11): 1357- 1367.

Wattenberg, L.W. (1981). Inhibition of carcinogen-induced neoplasia by sodium cyanate, tert-butyl isocyanate, and benzyl isothiocyanate administered subsequent to carcinogen exposure. Cáncer Research 41(8):2991-2994. 